\title{
Comparative aspects of in vitro proliferation of human and porcine intestinal cells exposed to mycotoxin zearalenone and its derivatives
}

\author{
I. Taranu ${ }^{1}$, D. E. Marin ${ }^{1}$, R. Burlacu ${ }^{2}$ and G. L. Calin ${ }^{1}$ \\ ${ }^{1}$ INCDBNA, National Institute of Research and Development for Biology and Animal Nutrition, Balotesti, Romania and \\ ${ }^{2}$ USAMV, University of Agriculture Science and Veterinary Medecine, Bucharest, Romania
}

Gastrointestinal epithelium provides a barrier against the mucosal penetration of feed and food contaminants and pathogens. In this in vitro study, we investigated and compared the cytotoxic effect of zearalenone (ZEN), mycotoxin that commonly contaminates cereals, and some of its derivatives: $\alpha$-zearalenol $(\alpha-Z \mathrm{ZOL}), \beta$-zearalenol $(\beta-\mathrm{ZOL})$, and zearalanone (ZAN) on three different intestinal cell lines. Intestinal cell lines from human (Caco-2 and Colo 320) or porcine (IPEC-1) origin, were cultured with increasing concentrations of ZEN, $\alpha$-ZOL, $\alpha$-ZOL and ZAN for 48 hours and cell cytotoxicity was measured by MTT [3-(4,5-dimethylthiazol-2-yl)-2,5-diphenyl tetrazolium bromide]. The dose response curves were fitted to a non-linear regression equations and inhibiting concentration of $50 \%$ (IC 50 ) were defined by the regression procedure using Corel QUATTRO ProX3, Mathcad Professional 14. For each mycotoxin multiple linear regressions (Corel QUATTRO Pro X3, Mathcad Professional 14) were used to establish correlations between human cells as dependent variable $(y$ or $z$ ) and porcine intestinal cells as independent variable $(x)$.

The results showed that increased concentrations of ZEN, $\alpha-Z O L, \beta-Z O L$ and ZAN decreased in vitro the cell viability in both species and cell types in a dose dependent manner, porcine IPEC cells being the most sensitive. The amounts of toxins necessary to inhibit viability, in a dehydrogenase enzyme activity assay, by 50\% were $113.0(\mathrm{ZEN}), 209.2(\alpha-\mathrm{ZOL}), 140.5(\beta-\mathrm{ZOL})$ and $81.6 \mu \mathrm{M}(\mathrm{ZAN})$ respectively in Caco-2 cells, $64.3(\mathrm{ZEN}), 105.9(\alpha-\mathrm{ZOL}), 38.2(\beta-\mathrm{ZOL})$ and $51.4 \mu \mathrm{M}(\mathrm{ZAN})$ in IPEC cells and 99.95 (ZEN), 142.4 $(\alpha-Z O L), 150.5(\beta-Z O L)$ and $215.4 \mu \mathrm{M}(\mathrm{ZAN})$ in Colo-320 cells.

Based on the results of this in vitro study, high mathematically correlations coefficients ( $\mathrm{r}$ between 0.939 and 0.990 ) were found between human (Caco-2) and pig (IPEC-1) intestinal cells for ZEN and its derivatives which suggest that pig could be used as a suitable model for comparison and for extrapolation to the human situations the effects concerning these mycotoxins. 